\title{
Scolia
}

Revue de linguistique

\section{L'asymétrie espace/temps dans les inversions locatives}

Space/Time Asymmetry in Locative Inversions

Catherine Fuchs

\section{(2) OpenEdition}

Édition électronique

URL : http://journals.openedition.org/scolia/875

DOI : $10.4000 /$ scolia. 875

ISSN : 2677-4224

Éditeur

Presses universitaires de Strasbourg

Édition imprimée

Date de publication : 11 juillet 2019

Pagination : 81-98

ISBN : 979-1-03440-050-8

ISSN : $1253-9708$

Référence électronique

Catherine Fuchs, «L'asymétrie espace/temps dans les inversions locatives », Scolia [En ligne],

33 | 2019, mis en ligne le 11 juillet 2019, consulté le 14 juillet 2019. URL : http://

journals.openedition.org/scolia/875; DOI : 10.4000/scolia.875 
SCOLIA 33/2019, p. 81-98.

\title{
L'asymétrie espace / temps dans les inversions locatives
}

\author{
Catherine FuCHS* \\ CNRS / ENS LATTICE, Université Paris 3 \\ catherine.fuchs@ens.fr
}

\section{Introduction}

Si l'inversion locative en général a fait l'objet de nombreuses publications (voir notamment Blinkenberg, 1928; Le Bidois, 1952; Jonare, 1976; Bresnan, 1994; Korzen, 1996; Fournier, 1997; Marandin, 1997 et 2003; Cornish, 2001 et 2005; Lahousse, 2011; Cappeau et Lahousse, 2015), en revanche la question de l'asymétrie espace / temps dans ce type de structure ne semble guère avoir retenu l'attention.

Soient les deux exemples suivants, qui correspondent respectivement à une inversion locative 'spatiale' et à une inversion locative 'temporelle' :

1) Derrière la place s'ouvre une esplanade. (Dépliant touristique)

2) En 1961 s'ouvre le procès Eichmann. (Le Journal du CNRS, 257, juin 2011)

La question est la suivante: les différences (à caractériser) entre ces deux exemples révèlent-elles une asymétrie entre le temps et l'espace?

Pour tenter de répondre, on examinera d'abord ce qui touche à la construction syntaxique $(\$ 1)$, puis on s'intéressera aux propriétés lexico-sémantiques des constituants $(\$ 2)$, avant de se pencher enfin

* Je remercie Anne Le Draoulec et Dejan Stosic pour leur relecture attentive d'une première version de cet article, ainsi que pour leurs suggestions judicieuses, qui ont permis de préciser certains points. 
sur les configurations interprétatives propres à chacun des deux types d'inversion locative $(\$ 3)$. Les différences observées nous conduiront à conclure à une asymétrie entre la localisation spatiale et la localisation temporelle. L'étude s'appuiera sur des exemples authentiques recueillis dans diverses sources contemporaines (presse, essais, ouvrages de vulgarisation, articles scientifiques, mémoires, fascicules touristiques).

\section{La construction syntaxique}

Les deux types d'inversion locative mettent en jeu un même schéma de base XVS; mais ce schéma commun se trouve investi différemment selon la nature de $\mathrm{X}$.

\subsection{Un même schéma de base XVS}

Dans les deux cas, le schéma XVS correspond à un noyau prédicatif, où la première position est occupée par un adverbial $\mathrm{X}$, la seconde par un verbe $\mathrm{V}$ (intransitif, réfléchi ou passif) et la troisième par un sujet $S$ postposé.

L'adverbial X fonctionne comme un complément intra-prédicatif (Fuchs, 2017) et occupe la première position du noyau (celle du sujet dans le schéma de phrase 'canonique' SVX), position qui permet de thématiser le constituant, d'où la dénomination de «topique scénique» souvent attribuée à X (Erteschik-Shir, 1997; Lahousse, 2011; Teixeira, 2016).

Le verbe $\mathrm{V}$ (intransitif, réfléchi ou passif) fonctionne de manière «inaccusative» (Mendikoetxea, 2006); il n'accepte pas de N complément d'objet direct (à l'exception des clitiques et des $\mathrm{N}$, généralement sans déterminant, qui participent du prédicat dans des locutions du type prendre tournure, entrer en application, retrouver vie, etc.).

Le sujet $S$ occupe la troisième position du noyau (celle de l'objet dans le schéma de phrase 'canonique' SVX), position d'un constituant rhématisé.

Le noyau XVS constitue un schéma de phrase complet (il n'accepte pas à la gauche de $\mathrm{X}$ d'élément périphérique de type circonstant) et autonome (on le trouve le plus souvent en indépendante, parfois dans une complétive), considéré globalement comme un constituant thétique. 


\subsection{Des exploitations différentes}

Pourtant, selon la nature de $\mathrm{X}$, des différences se manifestent, qui concernent la force du lien entre ce $\mathrm{X}$ et le reste de la relation prédicative. Trois ordres d'indices peuvent être invoqués.

\subsubsection{Les schémas à $X$ multiples}

Le premier indice syntaxique concerne les cas où le schéma d'inversion locative comporte plusieurs adverbiaux initiaux. Quand ces adverbiaux sont sémantiquement homogènes (plusieurs $\mathrm{X}$ spatiaux ou plusieurs X temporels), ils forment ensemble un constituant complexe qui occupe la première position du noyau - X2 venant simplement préciser X1:

3) Dans le palais Stabal, derrière l'Arsenal, se trouvait du temps des Habsbourgle Génie naval autrichien. (Magris, Déplacements, trad. fr. Brun, 2002 ; cit. in Le goût de la Croatie, 2005) $=$ «dans le palais Stabal, qui se trouve derrière l'Arsenal»

4) Peu de temps auparavant, en 1834, étaient nées les Études Symphoniques dont Clara donnera la première exécution publique "avec un courage d'homme», selon les termes de son futur époux. (Pochette CD-Rom de musique) $=$ "peu de temps auparavant, à savoir en 1834»

Quand les adverbiaux ne sont pas homogènes, ils correspondent à deux repères distincts ordonnés séquentiellement:

5) Il y a 4567 millions d'années, dans l'espace proche de ce qui deviendra le système solaire, explose une supernova. (Allègre, Introduction à une histoire naturelle, 1992)

6) Tous les ans, à Kortchoula, apparaissent au sommet de la tour, quadrillés à mi-corps par les hauts créneaux, deux être inconnus et cependant traditionnels, la tête ornée de couronnes antiques, l'un revêtu de noir, l'autre de pourpre. Et ces rois d'un passé lointain, qui surgissent ainsi, sans prévenir, de l'ancien décor, se redressent, saluent, comme avant un spectacle. (Domenach \& Pontault, Yougoslavie, 1960 ; cit. in Le goût de la Croatie, 2005)

7) En 1471, l'interminable conflit qui oppose les Yorks et les Lancasters pour le contrôle du royaume s'achève par la victoire des Yorks. [...] [Charles le Téméraire] pourrait donc tenter de relancer un conflit en se réclamant du trône d'Angleterre. Mais, 
au même moment, en France, se joue un autre enjeu dynastique qui le requiert aussi. (Le Monde, 31 juillet 2008)

Dans ce cas, c'est l'ordre X1(temps) $\rightarrow \mathrm{X} 2$ (espace) que l'on observe le plus souvent. S'il est clair que le $\mathrm{X}$ spatial (le plus près de $\mathrm{V}$ ) fonctionne bien comme un élément intra-prédicatif occupant la première position du noyau, en revanche le lien entre le $\mathrm{X}$ temporel (le plus éloigné de $\mathrm{V}$ ) et la suite du noyau SV s'avère plus distant: les adverbiaux temporels glissent en effet plus facilement vers l'extra-prédicativité que les adverbiaux spatiaux (qui, pour une partie d'entre eux, sont directement régis par $\mathrm{V}$ ).

\subsubsection{La virgule}

Le deuxième indice syntaxique concerne la présence possible d'une virgule entre $\mathrm{X}$ et la suite VS (schéma X,VS). Plusieurs ordres de raisons pouvant expliquer le recours à la virgule, nous excluons ici les cas de $\mathrm{X}$ longs et complexes pour ne considérer que les cas de $\mathrm{X}$ uniques, de forme relativement courte et simple:

8) En 2005, paraissait un rapport rédigé par dix inspecteurs généraux de l'Education nationale sur les signes et manifestations d'appartenance religieuse dans les établissements scolaires. (Finkielkraut, L'identité malheureuse, 2013)

9) Au mois de décembre, se tiendra à Copenhague une conférence internationale sur le climat qui scellera l'avenir de l'humanité (intertitre, Le Monde, 12 août 2009)

10) [à propos d'Eric Tabarly] Dix ans après sa mort, paraissent chez les libraires nombre d'ouvrages, au même moment qu'était inaugurée, à Lorient, la cité de la voile Eric Tabarly. (Le Monde des livres, $1^{\text {er }}$ août 2008)

On constate que, toutes choses égales par ailleurs, ce sont les $\mathrm{X}$ temporels qui sont plus souvent suivis d'une virgule, ce qui semble là encore indiquer un lien plus lâche au noyau prédicatif et une tendance à glisser vers l'extra-prédicativité. Comme l'ont noté Carruthers \& Le Draoulec $(2016, \$ 4)$, de tels adverbiaux détachés peuvent même, dans certains cas, fonctionner comme des «cadres de discours». 


\subsubsection{Les inversions absolues}

Le troisième indice syntaxique concerne un cas particulier d'inversion du sujet, appelé 'inversion absolue', où c'est V qui, en surface, se trouve à l'initiale de l'énoncé.

Deux types différents d'inversion absolue sont à distinguer, qui mettent en jeu des opérations très différentes. Le premier, que nous évoquons ici pour mémoire, est appelé inversion absolue 'élaborative'. Dans Sont admis les candidats qui ont la moyenne ou Est appelé isocèle un triangle qui a deux côtés de même longueur, la première position du noyau correspond à une place 'vidée', à laquelle se trouve identifié le sujet postposé S (schéma '( )VS', selon Fuchs, 2013); gloses: ceux qui sont admis sont les candidats qui [...], ce qui est appelé isocèle est un triangle qui [...].

Le second type, qui nous occupe ici, est, selon les auteurs, tantôt traité comme un cas particulier d'inversion locative (Lahousse, 2011: 79-81), tantôt décrit au contraire comme relevant d'un autre type d'inversion (Gournay, 2006). On le considèrera ici comme un schéma complexe construit sur la base du schéma d'inversion locative. Ce second type d'inversion absolue permet d'exprimer le surgissement brusque d'un événement ou d'un personnage: Arrive l'âge industriel; Vint une servante, qui prononça quelques mots; Entre Stolberg (didascalie) (exemples repris de Le Bidois 1952, I ch. 1, \$1-3). L'énoncécomporte en première position du noyau un constituant «zéro » $\varnothing$ (schéma ‘ØVS', selon Fuchs, 2013) - là où l'inversion locative classique comporte un adverbial X.

Ce constituant "zéro» marque une situation nouvelle, en rupture par rapport à une situation antérieure acquise et dépassée (Fuchs, 2016). Les inversions absolues de ce type se prêtent donc à une lecture temporelle, comme en témoigne la possibilité d'ajouter un adverbe temporel post-verbal (alors, ensuite ou enfin) co-indexé à la situation nouvelle marquée par $\varnothing$ : Arrive enfin l'âge industriel; Vint ensuite une servante, qui [...]; Entre alors Stolberg.

L'inversion absolue avec adverbe temporel post-verbal est attestée par de très nombreux exemples:

11) "[...] Je ne vous cacherai pas que, la première fois que je vous ai vu à Balbec, votre figure m'a été franchement antipathique." Suivaient alors des réflexions sur la ressemblance - remarquée 
le second jour seulement - avec un ami défunt pour qui $M$. de Charlus avait eu une grande affection. (Proust, cité par Le Bidois, 1952, I ch. 1, \$9)

$=$ « une fois ces propos énoncés (acquis d'une situation dépassée), suivaient alors $[\ldots] »$

12) En homme de la terre, [Alfred Courmes] fait d'abord capter l'eau de la source pour alimenter un puits, édifier les bâtiments agricoles, puis des terrasses pour créer un potager et un verger. Vient ensuite la conception du jardin d'agrément, structuré autour d'une imposante pergola. (Art et Décoration, 462, juilletaoût 2010)

$=$ «une fois ces travaux préliminaires effectués (acquis d'une situation dépassée), vient ensuite $[\ldots] »$

Dans les deux exemples ci-dessus, l'acquis de situation reste implicite. Mais il peut aussi être exprimé de façon explicite, dans une séquence périphérique au noyau (sorte d' «ablatif absolu»):

13) [Karine] souhaite "créer une maison d'hôtes pour citadins en manque d'air et de verdure». Elle fait appel à l'architecte Michel Courdouan pour diriger le chantier, qui durera un an. Une fois le gros auvre réalisé, arrive enfin l'étape de la décoration intérieure. (Art et Décoration, 446, octobre 2008)

14) Le requérant doit donc présenter des éléments de preuve [...]. Ces éléments pourront être de tout ordre mais certains d'entre eux sont susceptibles d'être particulièrement éloquents [...]. Une fois ces indices établis, pèsera alors une présomption simple de discrimination à l'encontre du défendeur. (Corpus ChambersLe Baron, 2006, LA-DF-EB-XX-02-02)

La lecture exclusivement temporelle de ce schéma d'inversion absolue constitue donc un troisième indice syntaxique de la différence entre l'espace et le temps dans les inversions locatives (ou apparentées): seul un repère initial temporel (renvoyant à une situation nouvelle en rupture par rapport à un acquis de situation) peut rester implicite (Ø).

\section{Les types lexico-sémantiques de constituants}

Regardons à présent ce qu'il en est de chacun des trois constituants du schéma XVS au plan lexico-sémantique. Ici encore, des disparités sont observables, selon qu'il s'agit d'une inversion locative spatiale ou temporelle. 


\subsection{Les types $d^{\prime}$ adverbiaux initiaux $X$}

Qu'il soit spatial ou temporel, l'adverbial initial X peut exprimer un repère ponctuel (absolu: à Paris, à midi; déictique: ici, aujourd'hui; relatif: plus loin, plus tard), un intervalle continu (fermé: de Paris à Marseille, de la naissance à la mort; borné à droite: jusqu'à la mer, jusqu'en 2017; borné à gauche: depuis le premier étage, depuis Noël) ou encore une progression (pas à pas, peu à peu).

Avec les adverbiaux déictiques/anaphoriques, des glissements interprétatifs sont possibles, principalement du temps vers l'espace. Des $\mathrm{X}$ intrinsèquement temporels sont couramment utilisés pour renvoyer à l'espace - qu'il s'agisse d'un espace concret:

15) L'appartement s'ouvre sur une entrée. Ensuite viennent le salon, puis la chambre. (Petite annonce immobilière)

ou d'un espace abstrait, notionnel:

16) L'Ordre des Templiers, très hiérarchisé, comprend tout d'abord les frères chevaliers. Puis viennent les prêtres et les clercs, ensuite les frères sergents et les écuyers et, enfin, la foule des valets et domestiques. (Sites Templiers et Hospitaliers du Larzac, Tourisme et culture en Aveyron, éditions du Beffroi, 2002)

Des exemples de ce type, où le parcours mental d'une série d'étapes spatiales statiques s'exprime à l'aide de $\mathrm{X}$ temporels et de $\mathrm{V}$ dynamiques, relèvent de la problématique plus générale de ce qu'on est convenu d'appeler le mouvement "abstrait» (Langacker, 1987) ou «fictif» (Talmy, 1999).

À l'inverse, il peut arriver que des $\mathrm{X}$ intrinsèquement spatiaux soient utilisés pour renvoyer à du temporel:

17) Maître Funakoshi écrira plusieurs livres sur le karaté [...]. Grâce à la contribution de nombreux élèves le premier dôjô de karaté est créé en 1938 [...]. C'est sans doute la période la plus heureuse de sa vie [...]. En 1945, lors d'un bombardement, le Shôtôkan est complètement détruit.

Ici débute une période très sombre pour maître Funakoshi car, peu près la destruction de son dôjô, sa femme meurt [...]. (<http://www.karate-kelmis.com/fr/karate-2/gichinfunakoshi/>, 19/12/2018)

Le Draoulec (2018), à qui nous empruntons l'exemple ci-dessus, note que l'interprétation temporelle du X peut être favorisée par le 
sémantisme du $\mathrm{N}$ (comme ici période), mais tient surtout au contexte discursif (présence d'une progression narrative et absence de référence spatiale dans le contexte gauche). Le glissement de l'espace au temps semble donc beaucoup plus contraint que le glissement du temps à l'espace.

\subsection{Les types de verbes $V$}

Certains V se rencontrent avec les deux types d'inversion locative: il s'agit de verbes de sens intrinsèquement dynamique; d'autres au contraire ne se rencontrent qu'avec l'inversion locative spatiale: ce sont les verbes statifs.

Se rencontrent avec les deux types d'inversion les $\mathrm{V}$ de sens dynamique marquant une phase initiale ou finale (apparaître, commencer, émerger, se former, s'établir, s'instaurer, etc.; se terminer, s'achever, etc.), ou bien un mouvement (arriver, entrer, défiler, venir, sortir, etc.). La nature (spatiale ou temporelle) du X peut cependant induire certaines modulations par rapport au sens intrinsèquement dynamique de ces verbes. Exemples:

18) [début d'un article] Ici commence ce voyage dans l'histoire des États-Unis, au carrefour de deux avenues, de la violence et de l'ennui, de la misère et de la liberté. Au tout début de cette Highway 61 d'où sont partis des millions de Noirs pour chercher dignité et fortune. (Le Monde, 11 août 2009)

$=$ «ici se situe le point de départ (spatial) de [...]»; commencer: sens statique de être à l'étape initiale

19) En 1939 commence la reconstruction du moulin. (Le Monde, 15 septembre 1989)

$=$ «en 1939 se situe le démarrage (temporel) de [...]»; commencer: sens dynamique de entrer dans sa phase initiale

20) Le lendemain, ma journée commence par un entretien avec les deux secrétaires, en compagnie de Sandrine. Au mur est affiché notre nouveau logo, grand format. C'est une réalisation de l'atelier Pastel pour les 50 ans du CAES: une réussite. Sur l'écran défilent de belles images. La Jordanie, où la Région a organisé un voyage [...]. (CAES Magazine 90, printemps 2009)

= "sur l'écran c'est un défilement de belles images qui passent en continu»; défiler: sens quasi-statique d'un déroulement continu, massif, compact (d'où défilement) 
21) Tel était le but de ce marathon qui s'est tenu du 16 au 18 mai, en ouverture du festival d'art qui envahit jusqu'au 15 juillet toute l'Islande. [...] Son principe? "Inventer un laboratoire, un parlement, qui réfléchisse à tout ce que nous voulons pour la société d'aujourd'hui. [...]». Durant ces deux jours défilent ainsi des équations, des performances, des projections, des milliards d'années, des artistes vétérans comme Jonas Mekas. (Le Monde, 12 juin 2008)

= «durant ces deux jours c'est un défilé de [...], qui se succèdent»; défiler: sens dynamique d'une itération ininterrompue de passages singuliers (d'où défilé)

Sont exclusivement employés dans l'inversion spatiale les $\mathrm{V}$ statifs (être, figurer, se situer, se trouver, etc.; passifs d'état: être placé, dissimulé, exposé, etc.):

22) Au centre du questionnement sur l'industrie nucléaire et les téléphones portables figure la problématique dite des "faibles doses", combinée à une exposition de longue durée. (Le Monde, 13 août 2008)

23) [La serre] est discrète et de proportions raisonnables. La preuve que l'on peut facilement installer une serre dans son jardin pour y cultiver sa passion. À l'intérieur se trouvent des pots, des outils de jardin, des paniers, des cloches en verre, qui font la vie d'un jardinier. (Art et Décoration, 450, avril 2009)

24) Conservée intacte, une fontaine, avec sa réserve d'eau sculptée dans la pierre, rompt la rectitude des murs de granit de cette ancienne ferme. Dans la niche improvisée sont exposés des bouteilles et des pots en grès. (Art et Décoration, 451, mai 2009)

Ces V statifs ne se rencontrent jamais avec l'inversion temporelle, qui comporte toujours des verbes dynamiques (passifs d'action: être organisé, être créé, etc.; événements ponctuels: éclater, se produire, etc.; activités graduelles: se développer, s'effacer, etc.):

25) Jacques Chirac inaugure sa nouvelle vie avec le lancement de sa fondation. L'ancien chef de l'État a tourné la page du pouvoir. Lundi 9 juin sera officiellement lancée sa fondation pour le dialogue des cultures. (Le Monde, 8-9 juin 2008)

26) Le 12 mai se produit le terrible tremblement de terre de Wenchuan, qui se solde par au moins 50000 morts et 5 millions de sans-abri. (Le Monde, 4 juin 2008) 
27) L'amour de l'Autre veille donc à ce que le présent ne sorte pas de soi. Et peu à peu s'efface, pour faire droit à la diversité, la contribution française à la diversité du monde. (Finkelkraut, L'identité malheureuse, 2013)

\subsection{Les types de noms sujets postposés $S$}

L'inversion spatiale tend à privilégier des $\mathrm{S}$ postposés renvoyant à des entités (humain, collectif d'humains, objet concret, entité abstraite, idéalité au sens de Flaux \& Stosic, 2015), plutôt qu'à des procès ou à des états:

28) Autour d'eux gravitent femmes et courtisans, opposants et rebelles, autant de "solitudes plurielles» dominées par une nature exotique et hostile qui demeure finalement seule juge de paix. (Le Monde, 15 mai 2009)

29) Derrière le «comptoir-bar» sont dissimulés les rangements et les appareils électro-ménagers. (Art et Décoration, 450, avril 2009)

30) Je connais Raguse intimement, j'y ai vécu en toutes saisons [...]. Sur chacune de ses places déferle une lumière symphonique, aussi riche en timbres que la plus libre fantaisie instrumentale. (T'Sertevens, Reflets, 1945 ; cit. in Le goût de la Croatie, 2005)

31) Là gît sans doute le grand mystère. Pour les scientifiques comme pour les autres. (Allègre, Introduction à une histoire naturelle, 1992)

32) À l'appui de cette thèse sont avancés des arguments ontogénétiques provenant des études du développement des aptitudes motrices chez l'enfant ainsi que de celles concernant la langue des signes. (Histoire, Épistémologie, Langage, XXIX: 2)

De son côté, l'inversion temporelle tend à privilégier des $\mathrm{S}$ postposés renvoyant à des procès (duratifs ou ponctuels) ou des états, ou encore à des mesures de temps (période, ère, moment, etc., cf. supra ex. 17):

33) Pendant six mois, d'octobre 2008 à mars 2009, sera organisée une concertation. Il en sortira un document-cadre, approuvé en conseil des ministres. (Le Monde, 22 août 2008)

34) En juillet 1976 s'est produit le tremblement de terre de Tangshan, qui a fait officiellement 240000 morts. (Le Monde, 4 juin 2008)

35) Puis s'établira un nouvel équilibre entre production d'énergie à l'intérieur et dissipation d'énergie à la surface. (Allègre, Introduction à une histoire naturelle, 1992) 
sans toutefois exclure des $\mathrm{S}$ renvoyant à des entités:

36) Sept années plus tôt s'était éteint Beethoven. (Pochette CD-Rom de musique)

37) Le $1^{\text {er janvier }} 2009$ entrera en application la réglementation européenne bio-révisée. (Le Monde, 10 juin 2008)

38) En mars dernier est sortie la $4^{e}$ version du navigateur Web Firefox. (Le Journal du CNRS, 257, juin 2011)

\section{Les configurations interprétatives}

Les différences syntaxiques et lexico-sémantiques observées entre l'inversion spatiale et l'inversion temporelle conduisent à proposer une typologie des configurations interprétatives auxquelles se prête chacun des deux types d'inversion locative. Comme on va le voir, ces configurations révèlent une asymétrie dans le mode de construction de la relation de localisation, selon la nature (spatiale ou temporelle) du repère $X$.

\subsection{L'inversion spatiale: la localisation d'une entité}

Dans le cas de l'inversion spatiale, la configuration prototypique correspond à la localisation d'une entité, localisation qui peut se faire par l'intermédiaire d'un $\mathrm{V}$ statif ou d'un $\mathrm{V}$ dynamique.

\subsubsection{Le repérage statique d'une entité: $X_{\text {spatial }} V_{\text {statif }} S_{\text {entité }}$}

Comme il a été dit plus haut, l'inversion spatiale tend à privilégier des $\mathrm{S}$ renvoyant à des entités, et elle seule permet de localiser ces $\mathrm{S}$ (c'est-à-dire de les repérer par rapport à $\mathrm{X}$ ) à l'aide de $\mathrm{V}$ statifs :

39) Plus loin que Louvres est un chemin bordé de pommiers dont j'ai vu bien des fois les fleurs éclater dans la nuit comme des étoiles de la terre: c'était le plus court pour gagner les hameaux. (Nerval, Sylvie, in Les filles du feu, 1854, rééd. 1994, chap. III) $=$ "plus loin que Louvres il $y a$ un chemin $[\ldots]$ »

40) La capitale, Kaboul, est déjà aux deux-tiers détruite, le pays est exsangue et les conditions de vie des Afghans auront vite fait de se détériorer plus encore à la suite des bombardements. Là se situe un autre défi de taille: le drame humanitaire qui a déjà commencé et qui ne manquera pas de s'accentuer, durant 
et après une attaque américaine. (Corpus Chambers-Le Baron, 2006, LA-AD-CD- XX-XX-01)

$=$ "là il y a un autre défi [...]»

Dans ces deux exemples, le $\mathrm{V}$ fonctionne comme un pur relateur quasiment dépourvu de potentiel sémantique propre, qui régit le $\mathrm{X}$ : il a pour seul rôle de repérer $\mathrm{S}$ par rapport au $\mathrm{X}$ spatial (être quelque part, se situer quelque part).

Dans d'autres cas, le $\mathrm{V}$ est porteur d'un certain potentiel sémantique et ne régit pas X; en plus de son rôle de relateur, il contribue alors aussi, de par son sémantisme propre, à qualifier $S$ :

41) Dans cette ville [Moncton] enneigée sept mois sur douze jurent encore quelques maisons noir ébène. (Le Monde 2, 8 août 2009) $=$ « dans cette ville il y $a$ encore quelques maisons noir ébène qui jurent»; jurer: sens statif de être dans un contraste détonnant (avec le blanc de la neige)

42) Sous le Grand Paris s'impose la question urbaine. (Le Monde, 17 octobre 2008)

$=$ «sous le Grand Paris il y a la question urbaine qui s'impose»; s'imposer: sens statif de être incontournable, ne pas pouvoir être évacué

La configuration prototypique de l'inversion spatiale se retrouve encore dans le cas de $\mathrm{S}$ renvoyant à des qualités, des propriétés ou des états, traités comme s'il s'agissait d'entités:

43) Je crois n'avoir jamais autant tenu à un livre qu'à mon manuel de lecture du cours élémentaire, Claude et Antoinette à la maison forestière. [...] Dans ce livre modeste tenait pour moi tout l'exotisme. (Ozouf, Composition française, 2009)

$=$ «dans ce livre il $y$ avait pour moi tout l'exotisme qui était contenu»

44) Dans la France de 1940, au chagrin de la défaite s'ajoutait pour moi l'angoisse de constater, çà et là, une certaine complicité avec elle: dans de larges zones croupissait une résignation hideuse, dénaturée. (Tillion, Fragments de vie, 2009) $=$ « dans de larges zones $i l$ y avait une résignation [...] qui croupissait» 


\subsubsection{Le repérage dynamique d'une entité: ' $X_{\text {spatial }} V_{\text {dynamique }} S_{\text {entité }}$}

On a vu que l'inversion spatiale admet aussi des V dynamiques. Le $\mathrm{V}$, ici encore, fonctionne comme le relateur qui participe au repérage de $S$, et dont l'apport sémantique propre vient qualifier l'activité de $S$ :

45) À côté du marché se forme un "public» qui lit, assiste aux spectacles et discute dans les clubs et les cafés. (Corpus ChambersLe Baron, 2006, LA-DF-BF-XX-01-01) = «à côté du marché il y a un "public» [...] qui se forme»

46) À côté de nous consommaient des Arabes, réfugiés par paquets sur les banquettes et qui somnolaient. (Céline, Voyage au bout de la nuit, cit. Damourette \& Pichon, EGLF, $\$ 1584$ ) $=$ «à côté de nous il y avait des Arabes [...] qui consommaient»

Dans la plupart des cas, $\mathrm{X}$ marque une localisation statique (comme à côté de dans les deux exemples ci-dessus). Dans d'autres cas, il constitue un repère directionnel, le $\mathrm{V}$ dynamique indiquant alors un mouvement de $S$ :

47) De Chantilly, de Compiègne et de Senlis accouraient de joyeuses cavalcades qui prenaient place dans le cortège rustique des compagnies de l'arc. (Nerval, Sylvie, in Les filles du feu, 1854, rééd. 1994, chap. IV)

48) Chez nous viennent tous les jours, avec dans leur sacoche le "midi» et le "quatre heures» [...], les enfants de cette énorme commune bocagère [...]. (Ozouf, Composition française, 2009)

$\mathrm{X}$ a alors une double fonction: il sert de repère pour la localisation de $S$ et il marque aussi le point de départ ou d'arrivée du mouvement de $S$; d'où les gloses possibles: "venant de Chantilly, de Compiègne et de Senlis, il y avait de joyeuses cavalcades qui accouraient», "chez nous il $\mathrm{y}$ a tous les jours les enfants [...] qui $y$ viennent».

\subsection{L'inversion temporelle: la localisation d'un procès}

Dans le cas de l'inversion temporelle qui, on l'a vu, comporte toujours un $\mathrm{V}$ dynamique, la configuration prototypique correspond à la localisation d'un procès par l'intermédiaire de ce V. Selon les cas, le procès peut être exprimé par le sémantisme de $\mathrm{S}$ ou par celui de $\mathrm{V}$. 


\subsubsection{Le repérage d'un procès nominalisé: ' $X$ temporel $^{V}{ }^{\text {(dynamique) }}{ }^{S}$ procès'}

Lorsque le procès est marqué par le $\mathrm{S}$, le $\mathrm{V}$ dynamique permet de repérer ce procès par rapport au $\mathrm{X}$ temporel et contribue au marquage du procès dans la mesure où il permet d'introduire une occurrence du procès nominalisé:

49) Le 25 janvier se produisent les premières tempêtes de neige dans le sud du pays [la Chine], phénomène très rare qui provoque une pagaille sans précédent dans les transports ferroviaires [...]. (Le Monde, 4 juin 2008) $=$ «le 25 janvier ont lieu les premières tempêtes de neige»

50) «Le matin, j’ouvre les serres pour les aérer. Puis vient l'arrosage. Ensuite, je n'ai pas de vrai programme.» (Le Monde, 3 avril 2012) $=$ "puis prend place l'arrosage»

Les expressions avoir lieu et prendre place figurant dans les gloses $\mathrm{du} \mathrm{V}$ ci-dessus sont proposées à dessein pour souligner le rôle de simple relateur localisant le procès $\mathrm{S}$ par rapport à $\mathrm{X}$. Des $\mathrm{V}$ comme se produire ou venir ne font en effet qu'actualiser (à la manière d'un 'verbe opérateur') le procès nominalisé dénoté par $S$; ils n'apportent aucun contenu sémantique spécifique. C'est pourquoi une paraphrase des exemples sous forme de phrase averbale serait possible: Le 25 janvier, premières tempêtes de neige; Puis: arrosage.

\subsubsection{Le repérage $d^{\prime}$ un procès-verbal : ' $X$ temporel $^{V}$ procès $S$ (entité)}

On a vu que l'inversion temporelle admet aussi des $\mathrm{S}$ renvoyant à des entités. Dans ce cas, c'est V seul qui marque le procès, cependant que l'entité $S$ désigne l'actant engagé dans ce procès:

51) Vers 85 naquit à Sinope, un port de la Mer Noire, un homme qui allait faire trembler l'Église des Pères apostoliques [...]. Cet homme a pour nom Marcion. (Marguerat, Le Dieu des premiers chrétiens, 2011)

$=$ «vers 85 eut lieu la naissance d'un homme qui $[\ldots]$ »

52) De temps à autre se réveillent les vieilles blessures du combat avec Tchouk, le coup de lance entre les côtes et le pouce fendu. (Deville, Peste et Choléra, 2012)

= «de temps à autre se produit le réveil des vieilles blessures [...]»

53) Dès 1963, Hanoï juge que le contexte est favorable au déclenchement d'une vaste offensive [...]. Mais les opérations sont reportées en raison de l'«américanisation» du conflit [...]. 
En 1966 émerge l'idée d'attaquer les grandes villes du Sud, ce qui fera toute l'originalité des opérations de 1968. (Le Monde 2, 31 mai 2008)

= «en 1966 se produit l'émergence de l'idée de [...]»

Le $\mathrm{V}$ dynamique joue ici un double rôle (que les gloses proposées ci-dessus visent à éclairer en le dédoublant): d'une part, en tant que relateur il actualise et localise le procès par rapport à X (d'où avoir lieu, se produire); d'autre part, en tant que lexème porteur d'un contenu sémantique, il exprime le procès dont $S$ est l'actant (procès restitué dans les gloses sous forme d'une nominalisation: naissance de, réveil de, émergence de). C'est pourquoi la paraphrase sous forme de phrase averbale est ici impossible: ${ }^{*}$ Vers 85 : un homme [...]; ${ }^{\star}$ De temps à autre, les vieilles blessures; ${ }^{\star}$ En 1965, l'idée de [...].

\section{Conclusion}

Nous voici en mesure de répondre à la question posée au début de l'article. En (54) (=1) et en (55) (=2), le verbe s'ouvrir s'interprète différemment du fait de l'asymétrie entre le repérage spatial d'une entité et le repérage temporel d'un procès (sans jeu de mot!):

54) Derrière la place s'ouvre une esplanade. (Dépliant touristique) = «derrière la place il y a / est / se trouve une esplanade qui s'ouvre»; s'ouvrir: sens statif de être (grand) ouvert

55) En 1961 s'ouvre le procès Eichmann. (Le Journal du CNRS, 257, juin 2011)

= «en $1961 \mathrm{il} \mathrm{y} \mathrm{a} \mathrm{/} \mathrm{a} \mathrm{lieu} \mathrm{/} \mathrm{se} \mathrm{produit} \mathrm{l'ouverture} \mathrm{du} \mathrm{procès}$ Eichmann»; s'ouvrir: sens dynamique de passer de non ouvert à ouvert

C'est pourquoi, si proches référentiellement que puissent sembler deux énoncés comportant le même verbe et le même sujet, mais introduits respectivement par un $\mathrm{X}$ spatial et par un $\mathrm{X}$ temporel, le repérage construit par l'inversion locative n'en est pas moins asymétrique:

56) À la Manufacture royale de Bains-les-Bains naquit en 1824 Julie-Victoire Daubié, première femme à décrocher le bac. (exemple forgé) $=$ «À la Manufacture royale de Bains-les-Bains il se trouva quelqu'un, à savoir J.-L. Daubié, qui naquit en 1824» 
57) Il y a 194 ans naissait Julie-Victoire Daubié, première femme à décrocher le bac. (Le Point, 26 mars 2018)

= «il y a 194 ans se produisait un événement, à savoir la naissance de J.-L. Daubié»

Il y a bel et bien asymétrie dans la façon dont se construit la localisation de la "scène» par rapport à $\mathrm{X}$, selon que l'on repère une entité dans l'espace ou un procès dans le temps. Localiser une entité par rapport à un repère spatial revient en effet à en prédiquer l'existence. Mais localiser un procès par rapport à un repère temporel ne constitue pas une prédication d'existence: qu'il soit exprimé par un verbe ou par un nom, le procès est déjà actualisé par les marques aspectuotemporelles portées par $\mathrm{V}$, en sorte que le repérage par rapport à $\mathrm{X}$ ne fait qu'apporter une précision supplémentaire. On comprend, dès lors, que les indices syntaxiques révèlent une plus grande distance entre $\mathrm{X}$ et la suite de la relation prédicative, dans le cas d'un X temporel.

\section{Bibliographie}

BLINKENBERG A. (1928), L'ordre des mots en français moderne, Copenhague, Bianco Lunos Bogtrykkeri.

BRESNAN J. (1994), Locative inversion and the architecture of Universal Grammar, Language 70, 72-131.

CAPPEAU P. \& LAHOUSSE K. (2015), Le sujet postposé, Encyclopédie Grammaticale du Français, <http://encyclogram.fr $>$.

CARRUTHERS J. \& LE DRAOULEC A. (2016), Encadrement discursif et postposition du sujet: l'éclairage des contes oraux, Journal of French Language Studies, (Peer reviewed version), Version en ligne (DOI: https://doi.org/10.1017/S0959269516000235).

CHAMBERS A. \& LE BARON F. (2006), Corpus d'articles de recherche en français (version électronique).

CORNISH F. (2001), L'inversion «locative» en français, italien et anglais: Propriétés syntaxiques, sémantiques et discursives, Cahiers de Grammaire 26, 101-123.

CORNISH F. (2005), A cross-linguistic study of so-called «locative inversion»: Evidence for the Functional Discourse Grammar model, in De Groot C. \& Hengevelt K. (eds.), Morphosyntactic expression in Functional Grammar, Berlin and New York, Mouton de Gruyter, 163-202. 
ERTESCHIK-SHIR N. (1997), The Dynamics of Focus Structure, Cambridge, Cambridge University Press.

FLAUX N. \& STOSIC D. (2015), Pour une classe des noms d'idéalités, Langue Française 185, 43-57.

FOURNIER N. (1997), La place du sujet nominal dans les phrases à complément prépositionnel initial, in Fuchs C. (éd.), La place $d u$ sujet en français contemporain, Louvain-la-Neuve, Duculot, $97-132$.

FUCHS C. (2013), L'inversion absolue en français: deux types d'absence à l'initiale, in Fesenmeier L. et al. (éds), L'absence au niveau syntagmatique, Frankfurt, Klosterman, 201-217.

FUCHS C. (2016), Une anaphore temporelle énigmatique: 'Arrive alors le temps de l'éméritat', in Sarda L., Vigier D. \& Combettes B. (éds), Connexion et indexation, ces liens qui tissent le texte, Lyon, Presses de l'ENS, 71-82.

FUCHS C. (2017), L'inversion locative revisitée: retour sur le statut du locatif initial, Verbum XXXIX: 2, 47-62.

GOURNAY L. (2006), Qu'est-ce qui distingue l'inversion absolue de l'inversion locative en français?, Lingvisticae Investigationes, 29: 1, 91-102.

JONARE B. (1976), L'inversion dans la principale non-interrogative en français contemporain, Stockholm, Almqvist och Wiksell.

KORZEN H. (1996), L'unité prédicative et la place du sujet dans les constructions inversées, Langue française 111, 59-82.

LAHOUSSE K. (2011), Quand passent les cigognes. Le sujet nominal postverbal en français contemporain, Paris, Presses Universitaires Vincennes.

LANGACKER R. (1987), Mouvement abstrait, Langue Française 76 : $1,59-76$.

LE BIDOIS R. (1952), L'inversion du sujet dans la prose contemporaine (1900-1950), Paris, d'Artrey.

LE DRAOULEC A. (2018), "Ici commence le court bonheur de ma vie»: ici temporel dans la narration, Langue française 197, 101116.

MARANDIN J-M. (1997), Dans le titre se trouve le sujet. Ou: l'inversion locative en français, Mémoire d'habilitation, Université Paris 7.

MARANDIN J-M. (2003), Inversion du sujet et discours dans les langues romanes, in Godard D. (éd.), Langues romanes. Problèmes de la phrase simple, Paris, éditions du CNRS, 345-392.

MENDIKOETXEA A. (2006), Unergatives that 'become' unaccusatives in English locative inversion structures: a lexical-syntactic 
approach, in Copy C. \& Gournay L. (éds), Points de vue sur l'inversion, Cahiers de Recherche en Grammaire Anglaise 9, Paris, Ophrys, 133-155.

TALMY L. (1999), Fictive Motion in Language and 'Ception', in Bloom P., Peterson M., Nadel L. \& Garrett M. (eds.), Language and Space, Cambridge M.A., MIT Press, 211-276.

TEIXEIRA J. (2016), Locative Inversion and Stage Topics: a crosslinguistic study, Discours XIX, <http://discours.revues. org/9229>; D.O.I.: 10.4000/discours9229. 